\title{
Personalized Recommendation System Based on WSN
}

\author{
https://doi.org/10.3991/ijoe.v12i10.6207 \\ Zhijun Zhang, Gongwen Xu, Pengfei Zhang and Yongkang Wang \\ Shandong Jianzhu University, Jinan, China
}

\begin{abstract}
Aiming at the limitation of the traditional user modeling technology in the personalized recommendation system, a new user modeling method based on wireless sensor network and ontology technology is introduced in this paper. In order to retrieve the tourist attractions conforming to the users' needs from a large amount of the tourist information, a personalized recommendation model based on the ontology technology and travel information is designed in this paper. Absorbing the context awareness research achievement, combining with the mobile Internet context sensitive features, the personalized recommendation algorithm based on ontology is discussed. The application research of the ontology technology applying in collaborative filtering recommendation system is analyzed deeply, and then the personalized and visual information push service delivery for users is realized. The experimental results show that the proposed WsnTRS recommendation system has a higher prediction accuracy comparing with the existing similar algorithms.
\end{abstract}

Index Terms-Location based Social Network, Ontology, Personalized Recommendation, Wireless Sensor Network

\section{INTRODUCTION}

In recent years, Wireless Sensor Network (WSN) is a new developed communications technology with the rapid development of communication technology, computer technology, sensor technology. The development of the WSN makes the vision of the Internet of things come true. WSN has more advantages than traditional RFID such as WSN is low cost, low power consumption, automatic management and high degree of cooperation [1]. With the continuous improvement of the smart phones, PDA, tablet PCs and other mobile intelligent terminals and mobile information environment, the user's behavior patterns are constantly changing, which enables a variety of information services of the mobile network environment to become a new area of potential growth and provides a wealth of contextual information for personalized recommendation. The range of mobile context perception not only includes the user's time, place, behavior, but also contain various type of sensor information and union information, etc. Through a comprehensive analysis of the range of mobile context perception, features and operation mechanism, we can take to analyze and predict its demand for knowledge services, so it can be better providing more comprehensive and reliable scenarios simulation for mobile information.

One of the key advantages of wireless sensor networks is their ability to bridge the gap between the physical and logical worlds, by gathering certain useful information from the physical world and communicating that information to more powerful logical devices that can process it. Wireless sensor network has a very important function, namely the tracking of mobile node. It is the actual application value that location information combined with a measured value. The common tracking in the node technology can be divided into range-based and range-free positioning according to the measurement method. Orientation method based on distance measurement technology measures the distance or angle between the mobile node and the reference node. Then it uses the localization algorithm such as trilateral and triangulation, maximum quasi likelihood estimation to compute nodes coordinates. Non ranging technology positioning does not depend on the distance and angle between the mobile node and the reference node. It realizes the positioning only according to the network connectivity information. Generally the positioning accuracy of ranging technology is rough.

The remainder of this paper is organized as follows. Section 2 describes the related work and application. In Section 3, we introduce related definitions and descriptions. In Section 4, we provide an overview of wireless sensor networks based travel personalized recommendation system. In Section 5, we describe the experimental results and analysis. Finally we conclude and discuss some future work in Section 6.

\section{RELATED WORK}

Recommender systems are software tools and techniques providing suggestions for items to be of use to a user. Collaborative filtering $(\mathrm{CF})$ recommendation algorithm is widely-applied information filtering technology in recommended systems. The specific algorithm classification is shown as Figure 1.

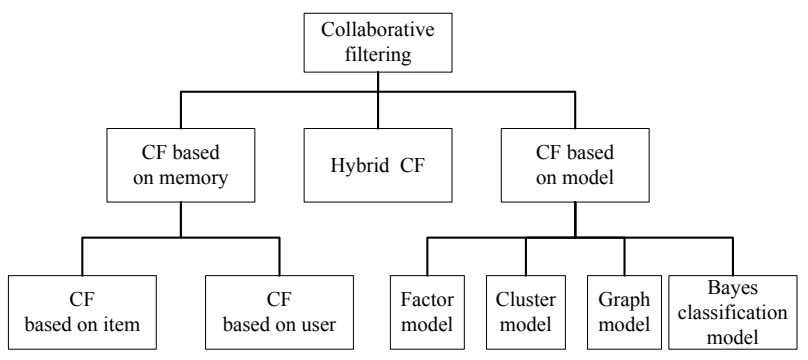

Figure 1. Classification of $\mathrm{CF}$ algorithm

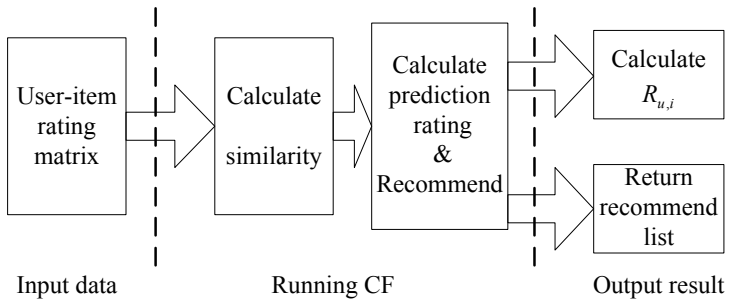

Figure 2. CF recommendation algorithm 


\section{SHORT PAPER \\ PERSONALIZED RECOMMENDATION SYSTEM BASED ON WSN}

Figure 2 shows a typical collaborative filtering recommendation process, which includes three stages, input-ting data stage, running collaborative filtering algorithm and outputting recommend results.

In recent years, there has been a lot of research about personalized recommendation and wireless sensor network. Ye etc. [2] explore user preference, social influence and geographical influence for POI (Point of Interest) recommendations. They argue that the geographical influence among POIs plays an important role in user check-in behaviors. In paper [3], an intelligent system to provide personalized recommendations of tourist attractions in an unfamiliar city is presented. Through tourism ontology, the system allows integration of heterogeneous online travel information. In paper [4] they propose a cocktail approach on personalized travel package recommendation. Specifically, they first analyze the key characteristics of the travel packages. Literature [5] presents a Bayesian model of mobility in populations that is not subject to any of these requirements. In paper [6] they compare different techniques found in the literature and study the characteristics of each one, highlighting their principal strengths and weaknesses. Gavalas etc. [7] propose the use of wireless sensor network installations around tourist sites for enabling precise localization and also providing mobile users convenient and inexpensive means for uploading tourist information and ratings about points of interest via their mobile devices. They also introduce the concept of 'context-aware rating', whereby user ratings uploaded through WSN infrastructures are weighted higher to differentiate among users that rate POIs using the mobile tourist guide application while onsite and others using the Internet away from the POI. In paper [8], they present a detailed survey on various trust models that are geared towards WSNs. Then, they analyze various applications of trust models. They are malicious attack detection, secure routing, secure data aggregation, secure localization and secure node selection. In paper [9], a Web-based system was developed that allows a user to mine the database using parameters such as the type of data, location of sensor, and the time of data acquisition. It is anticipated that this research will demonstrate the potential of using wireless sensor networks for monitoring buildings.

Zhang etc. [10] made a full discussion on the concept and research progress of mobile recommendation system. Many personalized service is based on the location information of the recommendation service. For example, the city's tourism services can sent information which is related to location to the phone, including the introduction of places of historic interest and scenic beauty, the tourist routes between the various scenic spots, which can help visitors to find the nearest restaurants, banks, bus station, hotels and other information from the current location[11]. In a large museum, visitors can use a voice guide, which can perceive the location to enjoy the explanation of the various collections [12]. Literature [13] is social networking services driven by GPS data that people can share the experience of life through share the historical position and make personalized friends recommendation. Literature [14] based on the use of location technology, considering the campus user's situation, and we can use the portable, wirelessly connected devices to construct X-ray glasses, so that each user can see the people and buildings nearby, which can facilitate the users to find friends, departments, laboratories or interesting activities nearby. Cantador etc. [15] proposed personalized information recommendation system News@hand, which based on the ontology. Using of semantic technologies in this system is to recommend the online news, Message content and user preference are described as concepts in domain ontology, and the context information of user preference is also considered. Deng etc. [16] proposed a context-aware system based on the need to combine with mobile terminals, context-awareness and wireless sensor networks, applied to mobile terminals. The system includes a number of wireless sensor nodes, situational awareness and processing terminals, application services and the implementation of the device, etc.

The existing recommendation algorithms are based on the similarity between users or items, and some recommendations are based on the user's social information, but all above algorithms don't really expose properties and characteristics of the commodity itself, that can't accurately recommend users interest in tourist routes and products. As for the problem, this paper draw on the research results of the scene sensitivity and combined with mobile Internet scene sensitive features. This paper discusses personalized recommendation algorithm based on ontology and indepth analyzes of the application of ontology technology in collaborative filtering recommendation system in order to provide users with personalized and visual information push service.

\section{RELATED DEFINITIONS AND DESCRIPTIONS}

\section{A. Ontology Concept}

Ontology, originated in the field of philosophy, is widely used in the fields of artificial intelligence, information retrieval, semantic web, natural language processing and so on[17]. Ontology has the characteristics of clarity, formalization and sharing.

Gruber first gave the definition of ontology, which is a formal description of the shared conceptual model [18]. In 1998, Guarino gave a concise definition of ontology and points out that ontology is a logical theory, which is used to illustrate intended meaning of a formal vocabulary [19].

The ontology formalization description is as follows:

$O=\left\{C, R, H_{C}, R \mathrm{el}, A_{o}\right\}$

Where, $C$ is the set of concepts.

$R$ is the set of relations.

$H_{c}$ shows the concept hierarchy, that is, the taxonomy re-

lation between concepts.

$\operatorname{Re} l$ shows the non-taxonomy relation.

$A_{o}$ is the ontology axiom.

\section{B. Architecture of Wireless Sensor Networks}

Wireless sensor network system architecture is shown in Figure 3. Wireless sensor network system usually consists of sensor nodes, sink nodes and management nodes. A large number of sensor nodes are randomly deployed in the monitoring area (sensor field) in order to form a network. The data collected by the sensor nodes is transmitted through other sensor nodes in the network, through the multi hop routing to the sink node, and finally the data through the Internet or satellite to the data processing center management node. 


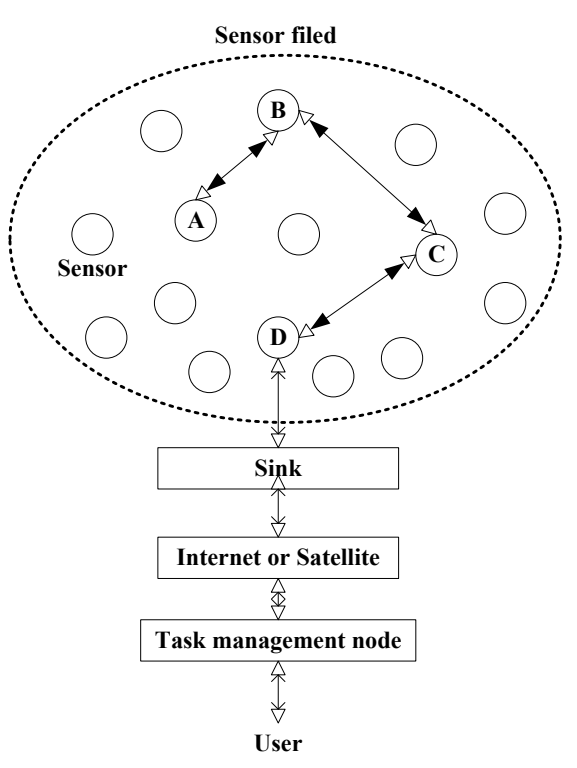

Figure 3. Wireless sensor network architecture

\section{Location-Based Social Networking (LBSN)}

LBSN is a kind of social network, in addition to the traditional social network of the connection among people, and LBSN can also track and share the location of other people's information. For example, you can use LBSN to collect the most trusted location recommendation, and take advantage of these recommendations' to sort the location by the degree of interest, in order to discover new POI (Point of Interest).

\section{WIRELESS SENSOR NETWORKS BASED TRAVEL PERSONALIZED RECOMMENDATION SYSTEM}

Firstly we study the current popular tourism personalized recommendation model in this paper, point out the shortcomings in these models and algorithms, and then put forward a travel personalized recommendation model WsnTRS which based on wireless sensor network. On the basic of utilizing the user's location and time information, the model digs into the depth of the potential users of social relations that can help users to find others who have the similar preferences, then to make corresponding recommendations according to mobile users of social relations, aiming to solve the problem of data sparsity and low recommendation accuracy in the field of personalized recommendation technology of electronic commerce.

\section{A. The Mobile Node Localization Algorithm based on Monte Carlo Method}

Currently, the main wireless localization technology includes infrared, wireless local area network, Bluetooth, ultrasonic, computer vision, and magnetic field, etc. The localization algorithm of wireless sensor networks carried out in this section is focused on how to realize the localization of mobile nodes in a more general network environment[20]. Using some previous positions of the nodes to carry on the interpolation, the approximate motion trajectory is obtained to predict the direction of motion of nodes.

The $k$ different time locations of the mobile node $N_{m}$ are: $\left(x_{0}, y_{0}\right),\left(x_{1}, y_{1}\right), \mathrm{L},\left(x_{k-1}, y_{k-1}\right)$. Now the location and motion direction of the node at the moment $k$ are predicted by the motion of the previous points.
When knowing the location of the previous $k$ moments, the location of the moment $k$ can be predicted using the method of three-dimensional interpolation. While considering the node's computing power and energy saving, the two dimensional Newton interpolation can be used. The interpolation of the $k+1$ moments $\left(x, t_{i}\right)$ and $\left(y, t_{i}\right)$ are computed respectively. Assuming $f(t)$ function value in $k+1$ different moments $t_{0}, t_{1}, \mathrm{~L}, t_{k}$ are $f\left(t_{0}\right), f\left(t_{1}\right), \mathrm{L}$, $f\left(t_{k}\right)$ respectively, that is $x_{0}, x_{1}, \mathrm{~L}, x_{k}$. According to the Newton interpolation polynomial, we can use the $f\left(t_{i}\right)$ of the previous $k+1$ moments, $X_{i}$, to predict $x_{k+1}$ of the $k+2$ moment. The formula is as follows:

$$
\begin{gathered}
x=f(t)=N_{k}(t)+R_{k}(t) \\
N_{k}(t)=f\left(t_{0}\right)+f\left[t_{0}, t_{1}\right]\left(t-t_{0}\right) \\
+f\left[t_{0}, t_{1}, t_{2}\right]\left(t-t_{0}\right)\left(t-t_{1}\right) \\
+\mathrm{L}+f\left[t_{0}, t_{1}, \mathrm{~L}, t_{k}\right]\left(t-t_{0}\right)\left(t-t_{1}\right) \mathrm{L}\left(t-t_{k}\right) \\
R_{k}(t)=f\left[t_{0}, t_{1}, \mathrm{~L}, t_{k}\right]\left(t-t_{0}\right)\left(t-t_{1}\right) \mathrm{L}\left(t-t_{k}\right) \\
y=g(t)=N_{k}^{\prime}(t)+R_{k}^{\prime}(t)
\end{gathered}
$$

From formula (1) and (4), we can predict the node velocity of $v_{x}$ and $v_{y}$ in $x$ direction and $y$ direction at $k+1$ moment are as following.

$$
\begin{aligned}
& v_{x}=f^{\prime}(t) \mid t=k \\
& v_{y}=g^{\prime}(t) \mid t=k
\end{aligned}
$$

The velocity of movement of the node is as follows.

$$
v_{\max }=\sqrt{v_{x}^{2}+v_{y}^{2}}
$$

So, the position of the mobile node $N_{m}$ at $k+1$ moment is as follows.

$$
\begin{aligned}
& x_{k+1}=\frac{1}{N} \sum_{i=1}^{N} x_{k+1}^{i} \\
& y_{k+1}=\frac{1}{N} \sum_{i=1}^{N} y_{k+1}^{i}
\end{aligned}
$$

\section{B. Construction of Tourism Domain Ontology}

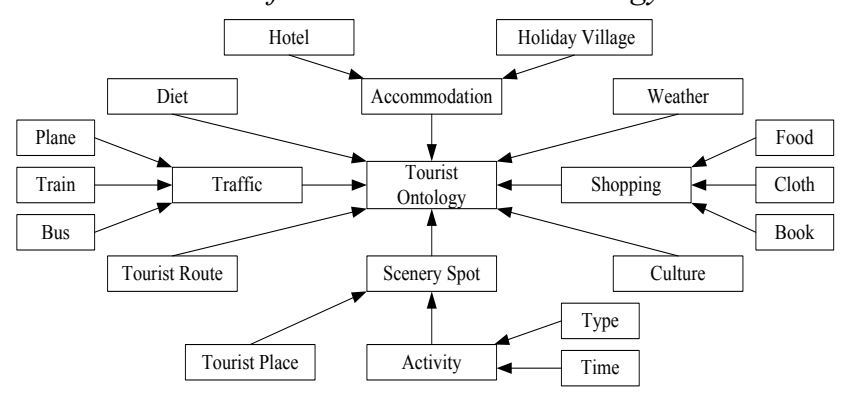

Figure 4. Diagram of tourist ontology

In this paper, the skeleton method is used to construct the knowledge ontology of tourism domain. First the ontology application scope and purpose are determined, analyzing the ontology knowledge. Secondly, the ontology is constructed. The Protégé software is used to realize the formal representation of the concepts and relations, and they are stored as OWL file. Finally, the established 


\section{PERSONALIZED RECOMMENDATION SYSTEM BASED ON WSN}

ontology and tourism personalized recommendation system are organically integrated. The established tourism domain ontology database is described as Figure 4.

\section{The Tourism Recommendation system based on WSN}

A tourism recommendation system WsnTRS based on wireless sensor network is constructed in this paper, which consists of three main modules: tourist information collection module, user modeling module and personalized recommendation module, as shown in Figure 5.

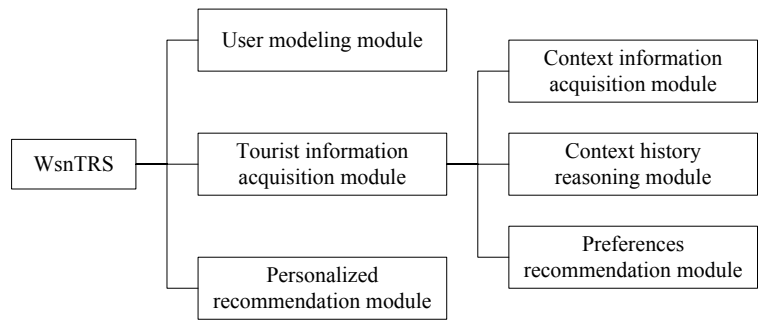

Figure 5. WsnTRS system modules

Comparing with the traditional information recommendation service based on Web, the tourism personalized recommendation system WsnTRS proposed in this paper realize the operation of perception, acquisition, transmission, storage, analysis and decision through wireless sensor network, and the data has the character of authenticity and space-time. This system can provide location and time sensitive personalized recommendation service to users. When users log in WsnTRS first time to register in the system, the file and contextual information are required to input. Secondly, using HUNC algorithm to mine the log files during 2015 Aug. $\sim$ Dec., the user interesting ontology is established based on the manual-constructed tourism ontology. Finally, a total of 18638 scenarios were collected. In the process of historical reasoning, 298 related rules are produced.

\section{EXPERIMENTAL RESULTS AND ANALYSIS}

\section{A. The influence of geographical location on the user's check-in behavior}

The characteristics of the user's check-in location and the users' social relationship are analyzed through the experiments. The number of check-in times is used as a function of the distance between the user's friend's and the user's location. The experimental results on the Foursquare, Brightkite and Gowalla data sets are shown in figure 4. From Figure 6, it can be seen that in the three data sets, the distribution functions are very similar, and they show the distribution of Power-Law functions. When the distance is less than $100 \mathrm{~km}$, the distribution is declined sharply. When the distance is more than 100 kilometers, the distribution is declined slowly.

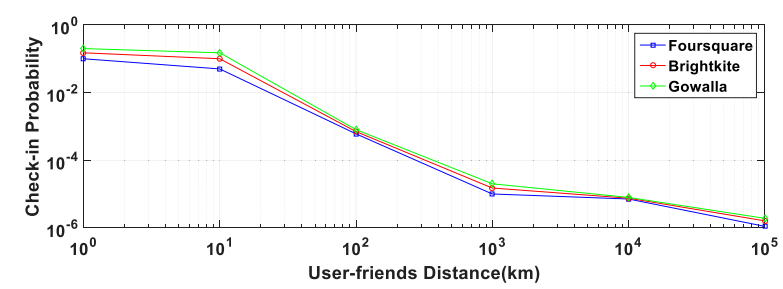

Figure 6. Relationships between the check-in probability and the user-friends distance
When two users are selected randomly, through the experimental results, the functions about the number of users check-in times and the distance of the random users location in the three data sets Foursquare, Brightkite and Gowalla are shown in Figure 7. From the figure, it can be seen that the distance between the user check-in probability and the random users do not present a complete decay pattern. When the distance is longer than $1000 \mathrm{~km}$, the check-in probability has a small increment, which is due to the uneven density of the population.

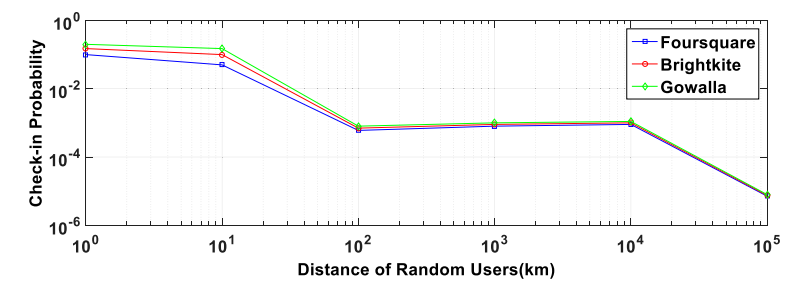

Figure 7. Relationships between the check-in probability and the distance of random users

\section{B. Recommended Performance Comparison}

\section{The Dataset}

An open source crawler Weblech is used to extract a tourism website travel information in this paper. The HTML documents of web pages are modified, then the information is converted into a DOM tree, removing the noise and filtering their relevant information, 12590 data were selected as the data set. Matching by using the ontology matching rules and the result of Chinese word segmentation, the obtained ontology concepts and their attributes are stored in the database. In order to collect the user's historical situation in formation, the user modeling module studied 200 mobile users' browsing behavior on the mobile site in the WsnTRS system.

\section{Comparison method}

In order to test the performance of the proposed WsnTRS model, the experiment is carried out to verify the validity of the model in this paper. Two recommendation algorithms are selected as the benchmark model: UserCF and ItemCF. Among them, the UserCF algorithm is based UserCF algorithms. This algorithm finds the similar users firstly, and then recommends commodities in accordance with the preferences of similar users. The ItemCF algorithm is based on ItemCF algorithms. It recommends similar commodities to target user based on the items 'similarities.

\section{Recommendation Accuracy Comparison}

In this paper, the data set is divided appropriately. The training set accounts for $10 \%, 20 \%, \ldots, 90 \%$, of the data set respectively. The prediction accuracy of WsnTRS algorithm, UserCF algorithm and ItemCF algorithm is shown in Figure 8. It is not difficult to see that, the prediction accuracy of WsnTRS recommendation algorithm is higher than UserCF and ItemCF regardless the division of the training set. In addition, the prediction accuracy of UserCF is higher than ItemCF. For new users, WsnTRS can infer their interest preferences from the association rules knowledge database based on context and user files, and then provide personalized tourism information. 


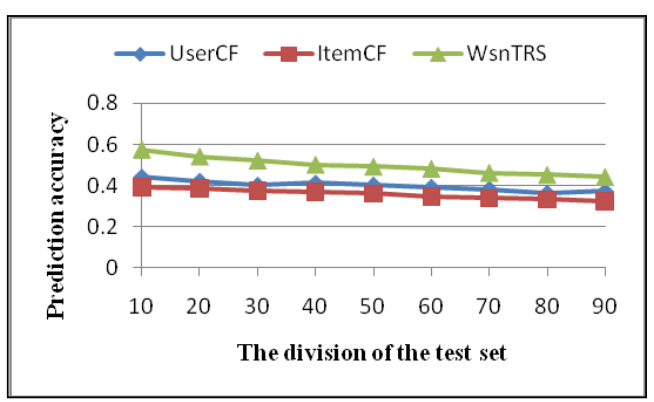

Figure 8. Performance comparisons of recommendation algorithm

\section{CONCLUSION AND FUTURE WORK}

In this paper, we research tourists tracking location management in the wireless sensor network environment in order to establish the foundation of the realization of the personalized travel recommendation system. This technical method provides the technical support for the personalized recommendation algorithm based on multi context. In this paper, we propose an intelligent mining system that based on the integration of context information and semantic ontology in a mobile Internet environment. There are some defects in this paper. The WsnTRS system is a comprehensive integrated system, and it needs for a full range of monitoring. We will continue to work on the integration of the system in the future work.

\section{ACKNOWLEDGMENT}

My deepest gratitude goes first and foremost to my cooperator. They have walked me through all the stages of the writing of this paper and have spent much of her precious time in offering valuable advice and guidance in my writing, and whose intellectual insights have contributed greatly to the completion of this paper. Without their contribution this paper could not have reached its present form. I would like to extend my heartfelt gratitude to the authors whose words I have cited or quoted, and to the scholars upon whose ideas I have freely drawn.

\section{REFERENCES}

[1] Senadeera P M, Dogan N S, Xie Z, et al. Recent trends in RFID transponders[M] //2013 Proceedings of IEEE Southeastcon. 2013.

[2] Ye M, Yin P, Lee W C, et al. Exploiting geographical influence for collaborative point-of-interest recommendation[C] //Proceedings of the 34th international ACM SIGIR conference on Research and development in Information Retrieval. ACM, 2011: 325-334. http://dx.doi.org/10.1145/2009916.2009962

[3] Huang Y, Bian L. A Bayesian network and analytic hierarchy process based personalized recommendations for tourist attractions over the Internet[J]. Expert Systems with Applications, 2009, 36(1): 933-943. http://dx.doi.org/10.1016/j.eswa.2007. $\underline{10.019}$

[4] Liu Q, Ge Y, Li Z, et al. Personalized travel package recommendation[C]//2011 IEEE 11th International Conference on Data Mining. IEEE, 2011: 407-416.

[5] McInerney J, Zheng J, Rogers A, et al. Modelling heterogeneous location habits in human populations for location prediction under data sparsity[C]//Proceedings of the $2013 \mathrm{ACM}$ international joint conference on Pervasive and ubiquitous computing. ACM, 2013: 469-478.

[6] Cacheda F, Carneiro V, Fernández D, et al. Comparison of collaborative filtering algorithms: Limitations of current techniques and proposals for scalable, high-performance recommender systems [J]. ACM Transactions on the Web (TWEB), 2011, 5(1): 2. http://dx.doi.org/10.1145/1921591. $\underline{1921593}$
[7] Gavalas D, Kenteris M. A web-based pervasive recommendation system for mobile tourist guides[J]. Personal and Ubiquitous Computing, 2011, 15(7): 759-770. http://dx.doi.org/10.1007/ s00779-011-0389-x

[8] Han G, Jiang J, Shu L, et al. Management and applications of trust in Wireless Sensor Networks: A survey[J]. Journal of Computer and System Sciences, 2014, 80(3): 602-617. http://dx.doi.org/10.1016/j.jcss.2013.06.014

[9] Jang W S, Healy W M, Skibniewski M J. Wireless sensor networks as part of a web-based building environmental monitoring system[J]. Automation in Construction, 2008, 17(6): 729-736. http://dx.doi.org/10.1016/j.autcon.2008.02.001

[10] Zhang X, Li C, et al. Evolution in knowledge services of big data: information mobile recommendation service model for big data [J]. Library \& Information, 2013, 157(04): 74-79.

[11] Tang K, Xu F, et al. Survey on location-based services [J]. Application Research of Computers, 2012, 29(12): 4432-4436.

[12] Zhou A, Yang B, et al. Location-Based services: Architecture and Progress [J]. Chinese Journal of Computers, 2011, 34(7): 11551171. http://dx.doi.org/10.3724/SP.J.1016.2011.01155

[13] Zheng Y, Wang L, Zhang R, et al. GeoLife: Managing and understanding your past life over maps[C]/The Ninth International Conference on Mobile Data Management (mdm 2008). IEEE, 2008: 211-212.

[14] Gruteser M, Grunwald D. Anonymous usage of location-based services through spatial and temporal cloaking $[\mathrm{C}] /$ Proceedings of the 1st international conference on Mobile systems, applications and services. ACM, 2003: 31-42.

[15] Cantador, I., Bellogín, A., \& Castells, P. (2008, December). Ontology-based personalised and context-aware recommendations of news items. In Proceedings of the 2008 IEEE/WIC/ACM International Conference on Web Intelligence and Intelligent Agent Technology-Volume 01 (pp. 562-565). IEEE Computer Society. http://dx.doi.org/10.1109/wiiat.2008.204

[16] Deng Z, Zhang J, et al. Design and Implementation of ContextAware system based on uSD [J]. Application Research of Computers, 2010, 27(1).

[17] Zhang Z, Liu H. Research on Ontology-based Literature Retrieval Model. Computer Modelling and New Technologies, 2014, 6(8):281-289.

[18] Gruber T R. A translation approach to portable ontology specifications[J]. Knowledge acquisition, 1993, 5(2): 199-220. http://dx.doi.org/10.1006/knac.1993.1008

[19] Eschenbach C, Grüninger M. Formal Ontology in Information Systems[M]. IOS Press, 2008.

[20] Wang Y. A research on the localization technology of wireless sensor networks [D]. Hefei: University of Science and Technology of China, 2007.

\section{AUTHORS}

Zhijun Zhang was born in Shandong, China, in 1973. He received his Ph.D. degree in School of Information Science and Engineering, Shandong Normal University. $\mathrm{He}$ is currently an associate professor of School of Computer Science and Technology, Shandong Jianzhu University. His recent research interests include personalized recommendation, E-commerce and machine learning. (email: zzjsden@163.com).

Gongwen Xu (corresponding author) was born in Shandong, China, in 1974. He works in School of Computer Science and Technology Shandong Jianzhu University, and received the Master's degree in 2005 from Shandong University. Now he is pursuing the Ph. D. degree in Shandong Normal University. His interesting filed is spam detection, image processing. (e-mail: xugongwen@sdjzu.edu.cn).

Pengfei Zhang was born in Shandong, China, in 1990. He received the B.S. degree in communication engineering from Jinan University in 2014. Currently, he is a M.S. candidate in School of Computer Science and Technolo- 


\section{PERSONALIZED RECOMMENDATION SYSTEM BASED ON WSN}

gy, Shandong Jianzhu University. His recent research interests include personalized recommendation and Ecommerce. (e-mail: zpfsdjzu@sina.com).

Yongkang Wang was born in Shandong, China, in 1994. He received the B.S. degree in information management from Shandong Jiaotong University in 2016. Currently, he is a M.S. candidate in School of Computer Science and Technology, Shandong Jianzhu University. His recent research interests include personalized recommendation and E-commerce.
m18366132486@163.com).

This paper is supported by the Science and Technology Development Planning of Shandong Province (2014GGX101011, 2015GGX101018), A Project of Shandong Province Higher Educational Science and Technology Program (J12LN31, J13LN11, and J14LN14) and Jinan Higher Educational Innovation Plan (201401214, 201303001). Submitted 03 September 2016. Published as resubmitted by the authors 16 October 2016. 